Research Article

\title{
Study on Design and Diamond Turning of Optical Freeform Surface for Progressive Addition Lenses
}

\author{
Yi Wei ${ }^{\circ},{ }^{1}$ Peng Zhai ${ }^{\circ},{ }^{1}$ Xiaoyi Chen, ${ }^{2}$ and Lei He ${ }^{1}$ \\ ${ }^{1}$ Associated Engineering Research Center of Mechanics \& Mechatronic Equipment, Shandong University, Weihai 264209, China \\ ${ }^{2}$ Suzhou Mason Optical Co., Ltd., Suzhou 215028, China \\ Correspondence should be addressed to Peng Zhai; zhaip@sdu.edu.cn
}

Received 12 August 2019; Revised 21 October 2019; Accepted 8 November 2019; Published 22 February 2020

Academic Editor: Stylianos Georgantzinos

Copyright ( 2020 Yi Wei et al. This is an open access article distributed under the Creative Commons Attribution License, which permits unrestricted use, distribution, and reproduction in any medium, provided the original work is properly cited.

Optical freeform surface components have attracted much attention due to their high degree of design freedom and small size. However, the design and processing difficulty of such components limit its wide application in optics industry. In recent years, diamond turning has been considered an efficient method for processing optical freeform surfaces, but the research on tool path generation of this processing method is not systematic. Progressive addition lens (PAL) is a typical optical freeform surface and is widely used to correct people's vision problems. Firstly, this paper introduces a method of designing PAL. Then, an optimized tool path generation method for diamond turning of the optical freeform surface is proposed, the equal angle method is used to select the discrete points, and a tool nose radius compensation method suitable for both slow slide servo (SSS) and fast tool servo (FTS) is adopted. Finally, the turning experiment is carried out with a single point diamond lathe, and a PAL surface with a roughness of $0.087 \mu \mathrm{m}$ was obtained. The power and astigmatism distributions were measured using a Rotlex freeform verifier to verify the rationality of the optical design.

\section{Introduction}

An optical freeform surface is defined as any nonrotationally symmetric surface or a symmetrical surface that rotates around an asymmetrical axis [1]. Such surfaces can be designed in any shape, and in most cases, these surfaces have submicron profile accuracy and nanoscale surface quality [2]. The advent of the freeform surface provides designers with wider design space, which can achieve higher optical utilization through smaller optical elements, reduce the number of optical components in the optical system, and improve the optical performance. These components have a large number of requirements in the aerospace, biomedical, and scientific industries. It also has a lot of daily applications, such as automotive lighting systems, F-theta lenses in laser printers, and mobile phone cameras [3, 4].

At present, the main methods of fabricating optical freeform surfaces include injection molded [5, 6], grinding and polishing [7], raster milling [8,9], and diamond turning (or tool servo turning) [10-13]. In practical applications, appropriate processing methods are selected according to the comprehensive factors such as the material and size of the workpiece. Recently, slow slide servo (SSS) and fast tool servo (FTS) have been extensively studied by scientific research institutes and applied to optical freeform surface fabricating. Compared with other methods, diamond turning has its unique advantages: the workpiece can usually be formed in a single process, low cost, and good form accuracy and surface finish. As early as 1983, Douglas of the University of Tennessee in the United States used an FTS device based on air-floating guides and linear motors to machine off-axis parabolic mirrors [14], which also made FTS technology truly used in optical freeform surface machining.

This study focused on the design and fabrication of typical optical freeform surface PAL. The curvature of the lens changes continuously from top to bottom, which can physically help the eye focus and provide a continuous vision from far to near. This kind of lens was originally used to correct presbyopia, and now it is also used to treat myopia of 
teenagers. The design of PAL is very complicated and difficult. There are two main design methods, one is to model in CAD software and simplify the lens design as a functional minimization problem $[15,16]$, and the other is a mathematical method that can directly express the surface with a series of formulas [17-21]. The first design method is relatively simple, but the processing of subsequent data is more complicated, and it is usually necessary to use the spline interpolation to obtain the machining coordinates. Compared with the first method, the second method has less accurate control of astigmatism distribution, but its modeling is more accurate, so this method is adopted in this study.

In this paper, the design method of PAL is firstly discussed, which is divided into meridian line design and surface construction. On this basis, a design example is given and used for subsequent machining. Then, a general tool path generation method for tool servo turning of optical freeform surfaces is proposed, which can provide more stable cutting motion. Finally, the tool path was generated for the designed lens surface, and the turning experiment was carried out in the way of point-to-point with a single point diamond lathe. The experimental results are tested and analysed, which indicate that the optical distribution of the fabricated lens was conformed to the design principle, and the surface quality was good.

\section{Design of the PAL}

As people age, the crystalline lens becomes rigid and the flexibility of the ciliary muscle decreases. In order to see the close target clearly, they have to move far away, so presbyopic glasses need to be worn. Traditional presbyopic glasses cannot take into account both far and near sightedness. At this time, the bifocal lens was invented, but the visual effect of the glasses is not good because of its special structure. PAL can meet the needs of distant and near vision and provide a continuous field of view, so it is more comfortable to wear.

The functional partition of the PAL is shown in Figure 1. Area $A$ is the far-view zone, which is mainly responsible for distant vision. Area $C$ is the near-view zone, which mainly meets the needs of reading and other near-sighted objects. Area $B$ is a progressive corridor, which is the middle area connecting the far and near-sighted areas. This part can provide smooth and continuous vision, which is the focus of the design. Area $D$ is the blending zone, in which there is inevitable astigmatism. In this study, the design of the meridian line determines the vertex power distribution. Then, the designed surface was constructed according to the distribution of curvature along the line. This method can choose a large number of parameter combinations, and different parameters will generate different optical power and astigmatism distribution to meet the different needs of wearers.

2.1. Design of Meridian Line. The refractive ability of ophthalmic lens is generally expressed by the vertex power, which is the reciprocal of the paraxial focal length of the lens measured in meters. Its unit is diopter and its symbol is " $D$ ". It can be expressed by the following formula:

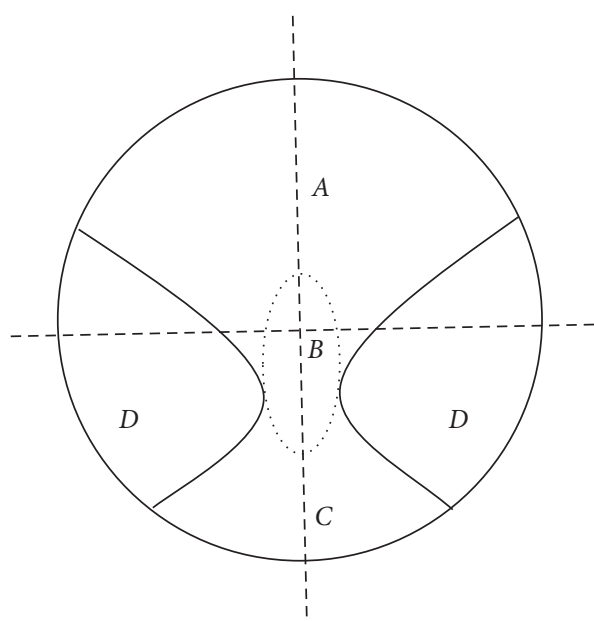

Figure 1: Functional partition of the PAL.

$$
D=1000 \times\left(\frac{n-1}{r_{1}}+\frac{1-n}{r_{2}}\right),
$$

where $n$ is the refractive index of the material and $r_{1}$ and $r_{2}$, respectively, represent the curvature radius of the front and back surfaces. From this formula, we can know that the vertex power of the glasses is the sum of the vertex power of the front and back surfaces. The difference between PAL and traditional lenses is that the curvature of one of its surfaces is variable. If the surface of curvature change is the front surface, it is called the exterior progressive addition lens, and if it is the back surface, it is the interior progressive addition lens.

As shown in Figure 2, $p p^{\prime}$ is the meridian line. The change in vertex power along the meridian line is called the addition power curve. In order to ensure smooth and continuous visual effect, the meridian line design should ensure the following: firstly, stable optical effect around distant-sighted point $F$ and near-sighted point $N$; secondly, smooth and gradual change of the power addition curve $D(x, 0)$ in the whole power-adding range. The region where the optical effect is stable means the region where the vertex power does not change significantly. In the optical design, the size of the stable region near $F$ or $N$ depends on the curvature of the surface (the vertex power is proportional to the surface curvature). The slower the curvature growth rate is, the larger the stable far-sighted or nearsighted area is.

In order to meet the abovementioned conditions, we need the curvature of the surface along the meridian line to change as smoothly as possible. As a criterion of smoothness, we can require that the mean square gradient of $D(x, 0)$ is the minimum, or in other words, that $D(x, 0)$ minimizes the Dirichlet integral [21]:

$$
\int_{F}^{N}\left|\frac{\mathrm{d}^{m+l-1} D(x, 0)}{\mathrm{d} x^{m+l-1}}\right|^{2} \mathrm{~d} x,
$$

where $m$ is the first order to make $\left(\mathrm{d}^{n}[D(x, 0)] / \mathrm{d} x^{n}\right)$ not zero at the distant-sighted point and $l$ is the first order to make $\left(\mathrm{d}^{n}[D(x, 0)] / \mathrm{d} x^{n}\right)$ not zero at the near-sighted point. And set the coordinates of the distant-sighted point as 


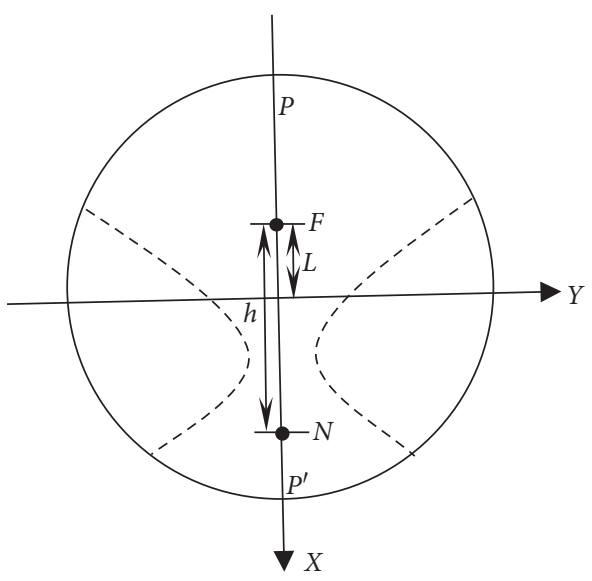

Figure 2: Diagram of PAL in $X Y$ plane.

$(-L, 0)$ and the distance between the distant-sighted point and the near-sighted point as $h$.

In order to obtain the minimum value of the abovementioned formula, according to Euler-Lagrange equation, the following results are obtained:

$$
\frac{\mathrm{d}^{m+l} D(x, 0)}{\mathrm{d} x^{m+l}}=0 .
$$

In order to make the curvature change slowly at the $F$ and $N$ point, the order of the first nonzero order derivative should be as high as possible. Solving equation (3) with boundary conditions

$$
\left\{\begin{array}{l}
D(-L, 0)=D_{F} \\
D(h-L, 0)=D_{N}
\end{array}\right.
$$

The following formula can be obtained:

$$
D(x, 0)=D_{F}+\left(D_{N}-D_{F}\right) \sum_{i=1}^{m+l-1} c_{i}(x+L)^{i}
$$
obtain

Substituting $D(h-L, 0)=D_{N}$ into equation (5), we can

$$
\sum_{i=1}^{m+l-1} c_{i} h^{i}=1
$$

According to the definition of $m$, we can obtain

$$
\left.\frac{\mathrm{d}^{i} D(x, 0)}{\mathrm{d} x^{i}}\right|_{x=-L}=0, \quad i=1,2,3, \ldots, m-1 .
$$

Equation (7) can also be written as follows:

$$
c_{i}=0, \quad i=1,2,3, \ldots, m-1,
$$

and equations (5) and (6) can be written as follows:

$$
\begin{aligned}
& D(x, 0)=D_{F}+\left(D_{N}-D_{F}\right) \sum_{i=m}^{m+l-1} c_{i}(x+L)^{i}, \\
& \sum_{i=m}^{m+l-1} c_{i} h^{i}=1 .
\end{aligned}
$$

According to the definition of $l$, the following formula can be obtained:

$$
\left.\frac{\mathrm{d}^{i} D(x, 0)}{\mathrm{d} x^{i}}\right|_{x=h-L}=0, \quad i=1,2,3, \ldots, l-1 .
$$

The coefficient $c_{n}$ can be obtained by solving the linear equations with the combination of equations (10) and (11), and the power addition curve can be obtained by choosing the appropriate parameters.

2.2. Construction of PAL Surface. After determining the vertex power distribution along the meridian line, we should extend the obtained power to the whole lens surface, and then calculate the surface vector height according to the principle of differential geometry. There are many ways to extend the power of the meridian to the whole lens surface. Generally, the contour line of equal power which is orthogonal to the meridian line is chosen [22]. The contour line can be straight line, circle, hyperbola, ellipse, and so on. The designer can select the contour line according to the actual needs.

The PAL shown in Figure 3 is externally progressive, meaning that this lens has a freeform front surface and a spherical back surface. In order to obtain the lens height, we assume that the lens is tangent to the vertical plane at point $O$. Make a horizontal plane perpendicular to the vertical plane and through point $O$, dividing the progressive surface into two parts along the meridian.

We define a Cartesian coordinate system in which the progressive surface is tangent to the $X O Y$ plane at point $O$, the $X$-axis is vertically downward, and the $Z$-axis points to the eye. As shown in Figure 4 , let $Q$ be any point on the meridian line, and the curvature radius of this point is $r$. Take a point of $q$ in the normal direction of the $Q$ point and make Qq equal to $r$. The $q$ point is the curvature centre of the $Q$ point. The trajectory of curvature centre at each point in the meridian is called the evolute of the meridian line, which is represented by $\mathrm{mm}^{\prime}$ in Figure 4 .

Let the coordinate of point $q$ be $(\varepsilon, \eta, \zeta)$, and the coordinate of point $Q$ on the meridian line $P P^{\prime}$ be $(u, 0, z)$, where $u$ represents the $X$ coordinate of $Q$ point. Then, the coordinates and curvature radius of $q$ point can be expressed by the equation containing $u$ :

$$
\left\{\begin{array}{l}
\varepsilon=\varepsilon(u), \\
\eta=0, \\
\zeta=\zeta(u), \\
r=r(u) .
\end{array}\right.
$$

According to the geometric relationship shown in Figure 4, the height $Z$ of $Q$ point relative to the $X O Y$ plane can be derived from the following formula:

$$
z=\zeta(u)-\sqrt{r^{2}(u)-[x-\varepsilon(u)]^{2}-[y-\eta(u)]^{2}},
$$

where 


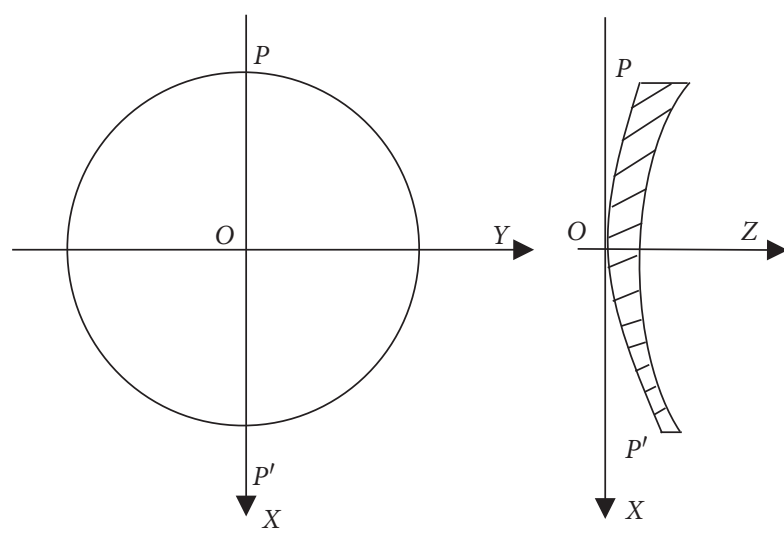

FIGURE 3: Structural sketch of lenses.

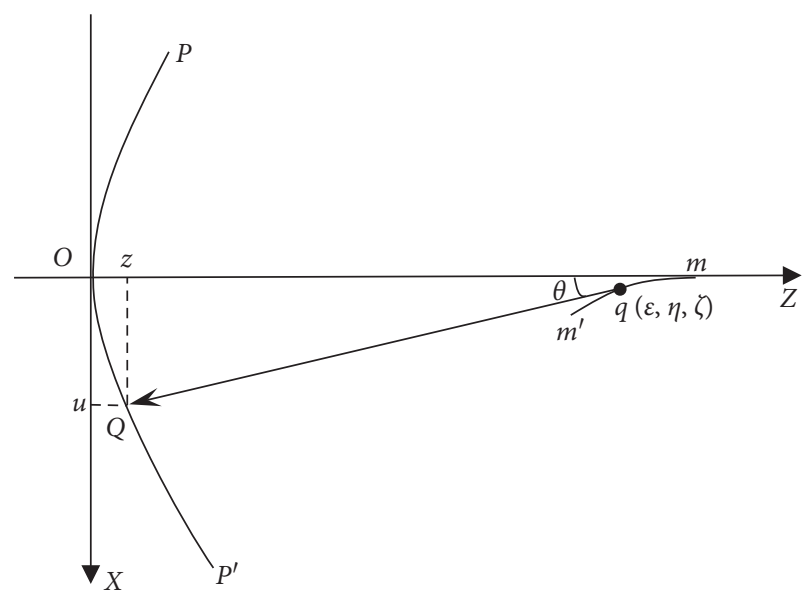

Figure 4: Schematic diagram of the progressive surface structure.

$$
\left\{\begin{array}{l}
\varepsilon(u)=u-r(u) \sin \theta(u), \\
\eta(u)=0, \\
\zeta(u)=r(u) \cos \theta(u)+\int_{0}^{u} \tan \theta(u) \mathrm{d} u, \\
\sin \theta(u)=\int_{0}^{u} \frac{1}{r(u)} \mathrm{d} u .
\end{array}\right.
$$

According to the formula for calculating the vertex power of Section 2.1, if the externally progressive design is adopted and the curvature radius of the back surface of the lens is selected, combined with equation (9), the curvature radius $r(u)$ of any point on the meridian line can be expressed as follows:

$$
\frac{1}{r(u)}=\frac{1}{r_{F}}+\left(\frac{1}{r_{N}}-\frac{1}{r_{F}}\right) \sum_{i=m}^{m+l-1} c_{i}(u+L)^{i}
$$

To sum up, the appropriate parameters (including $m, l$, $h$, and $L$ ) are selected according to the design requirements, and then the coefficient $c_{i}$ is solved by the linear equations, and the equation of the power addition curve can be obtained. The vector height equation of the surface can be deduced by selecting the appropriate contour curve $u(x, y)$ and substituting it into equations (13)-(15).

2.3. Design Example. A PAL surface was designed by the abovementioned method, assuming that the diameter of the lens was $70 \mathrm{~mm}$. The type of the contour curve that is orthogonal to the meridian line is selected as hyperbola. And the remaining design parameters can be seen in Table 1 . The final image of lens vector height is shown in Figure 5.

\section{Tool Path Generation for Diamond Turning}

The generation method of ultraprecision tool paths depends on the surface description. However, no matter the surface is modeled in CAD software or expressed directly by the mathematical formula, it must be transformed into point coordinates for processing. From Section 2, we have obtained the vector height formula of the PAL surface, and the next step is to generate the tool path suitable for diamond turning.

3.1. Diamond Turning Principle. A typical machine tool configuration for SSS technology is shown in Figure 6. The workpiece is mounted on the spindle and rotates with the spindle. The spindle is mounted on a working table with $X$ direction guide rail, and $Z$ direction guide rail drives the diamond cutting tool to complete reciprocating cutting motion. Sometimes, a fiber-linked probe was integrated onto the machine tool for the purpose of alignment [23]. The machining principles of FTS and SSS are similar,the difference is that the $Z$ direction cutting motion in the FTS technology is realized by the FTS device. This type of machine tool is distinguished from a conventional machine that can only fabricate rotationally symmetrical surfaces due to its special configuration. Any three-dimensional tool path of nonrotational symmetric freeform surface can be realized when the $X$-axis, $Z$-axis, and $C$-axis move simultaneously according to a given set of numerical motion commands [24].

It can be known from the above that diamond turning has three processing parameters: spindle angle, $X$ direction displacement, and $Z$ direction displacement. These three parameters, respectively, correspond to the machine tool's spindle, $X$-slide and $Z$-slide (or FTS). In order to generate motion commands for machining, we need to switch from the Cartesian coordinate system to the cylindrical coordinate system. Specifically, the $Z$ coordinates are the same in both coordinate systems, and Cartesian $(x, y)$ are converted to cylindrical $(\rho, \theta)$ by the following formula:

$$
\left\{\begin{array}{l}
x=\rho \times \cos (\theta) \\
y=\rho \times \sin (\theta)
\end{array}\right.
$$

3.2. Selection of Discrete Points. The turning path of the freeform surface is a spiral in space. First, we need to select a set of discrete points on the XOY plane, and then map these 
TABLE 1: Design parameters of PAL.

Power of far-view zone (D)

Addition power (D)

Length of progressive corridor: $h(\mathrm{~mm})$

The coordinates of the $F$ point: $L(\mathrm{~mm})$

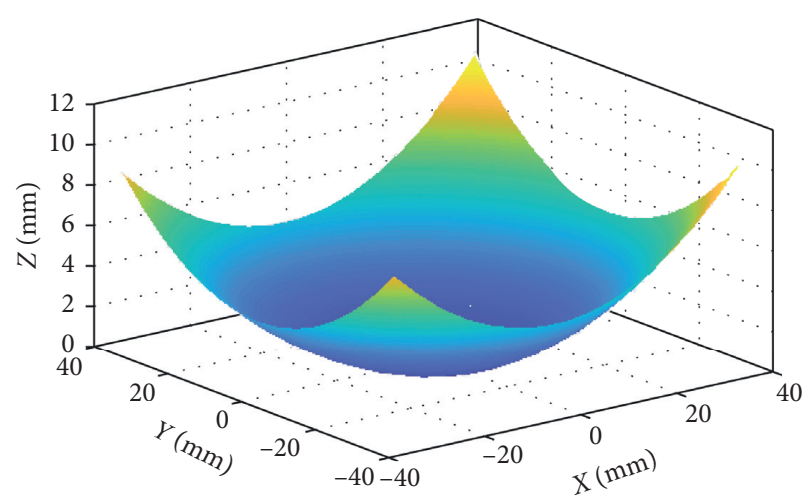

FIGURE 5: Lens vector height map.

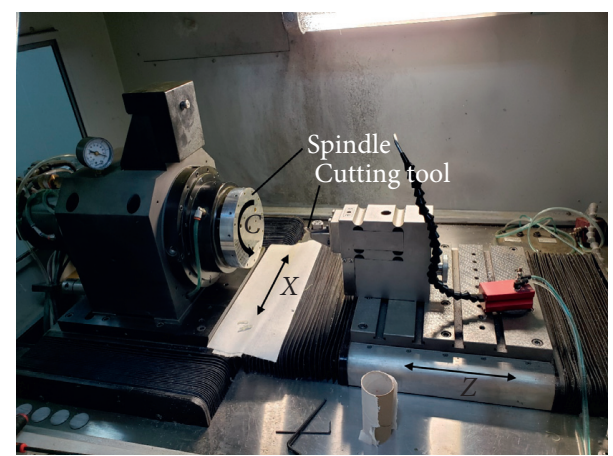

FIgURE 6: Photo for the SSS diamond turning machine tool.

points to the freeform surface to get the $Z$ direction coordinates, so as to obtain all the required processing data. There are three main methods to select discrete points: equal angle method, equal arc length method, and equal chord length method. In recent years, some scientific research institutes have put forward some variable feed methods, such as equal residual height method and equal load method, but the application is not extensive. The equal angle method means that, at the same turn of a plane helix, the central angle between any adjacent two points is equal. Similarly, the equal chord length method means that the two adjacent points have the same chord length; equal arc length rule means equal radians.

However, the time interval between cutting points selected by the equal chord length method and equal arc length method is not uniform. This leads to the need to configure time parameters at each cutting point in the turning process, which will greatly increase the amount of data in the machining file. Besides, these two methods will cause unnecessary spindle acceleration in the cutting process, which will affect the processing quality. Therefore, these two methods are not commonly used in the current research. In contrast, the equal angle method is simple to calculate, and the rotation angles of the spindle at the same time interval are the same, which does not produce redundant acceleration. It has been widely used in FTS and SSS processing, and often achieved better results.

In this paper, the equal angle method is used to select discrete points. The PAL surface designed in Section 2.3 was planned to generate discrete point graphs. In order to facilitate the demonstration, one point is taken at $10^{\circ}$ intervals and the feed rate is $5 \mathrm{~mm} / \mathrm{r}$, as shown in Figure 7. The $Z$ coordinates corresponding to each cutting point can be obtained by mapping the points on the XOY plane to the freeform surface.

3.3. Tool Nose Radius Compensation. Optical freeform machining generally adopts the diamond arc edge turning tool, which can process workpiece of various shapes and obtain high surface quality, and has been widely used in precision machining. However, in the process of machining, the radius of tool nose will lead to over cut and affect the surface fidelity. For the optical freeform surface, the precision of the surface shape directly affects its optical capability, so the compensation of the tool radius cannot be ignored. The influence of tool nose radius is shown in Figure 8. The dotted line represents the contour of the ideal surface and the solid black arc represents the contour of cutting edge. When the radius of the tool nose is not compensated, the cutting point in the tool path is determined by the position of the tool tip. It can be seen from the figure that the round tool nose geometry will lead to over cut.

At present, the tool nose radius compensation method adopted by most scientific research institutions is the twoway tool radius compensation [11,25], in which the cutting point position is determined in advance, and the radius compensation is then carried out in the normal direction of the cutting point. The feature of this method is that at different cutting points on the freeform surface, the shift value is not constant as the slope angle changes [24]. Hence, it can make the tool vibrate in the radial direction, which requires high performance of $X$-slide. It may not be able to compensate when machining very complex microstructures, and it is not suitable for single-degree-of-freedom FTS processing. In this paper, a one-way tool nose radius compensation method is adopted, which only compensates in $Z$ direction and makes $X$ feed free of vibration. Moreover, it is suitable for both SSS and FTS technology.

According to the diamond turning principle of the freeform surface, the motion of the tool in the cutting process is completed on the $X O Z$ plane, which can be expressed as $\rho=x$ in the cylindrical coordinate system. When using the one-way compensation method, the radial coordinate only depends on the cutting radius and the feed rate. This tool nose radius compensation method is illustrated in Figure 9.

The curve $L$ is the transversal of the freeform surface on the $X O Z$ plane, $C$ is the tool contact point, $S$ is the tool location point, and $a_{f}$ points to the direction of tool feed. In the process of machining, the tool location point $S$ is uniformly fed, so we can determine the radial coordinates of this 


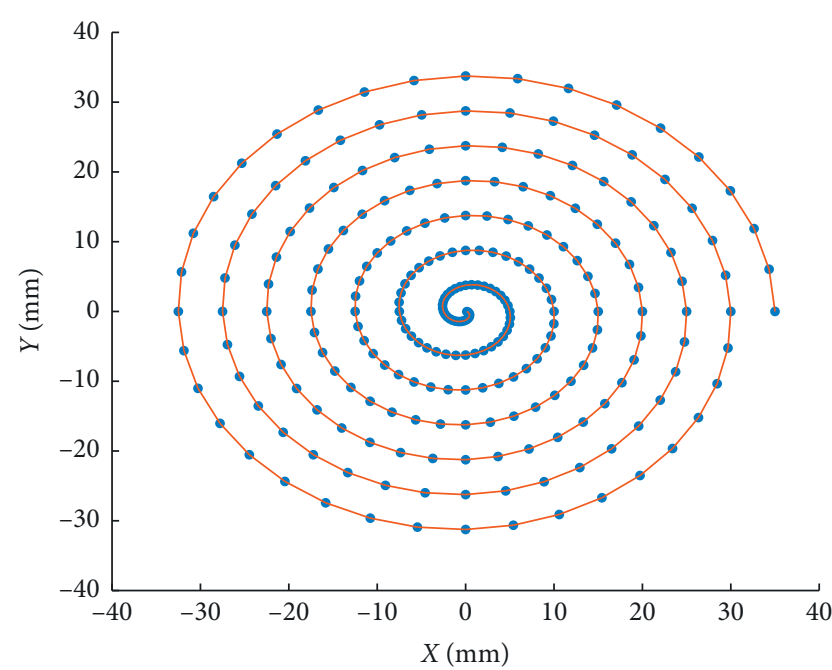

FIGURE 7: Schematic diagram of discrete points of the XOY plane.

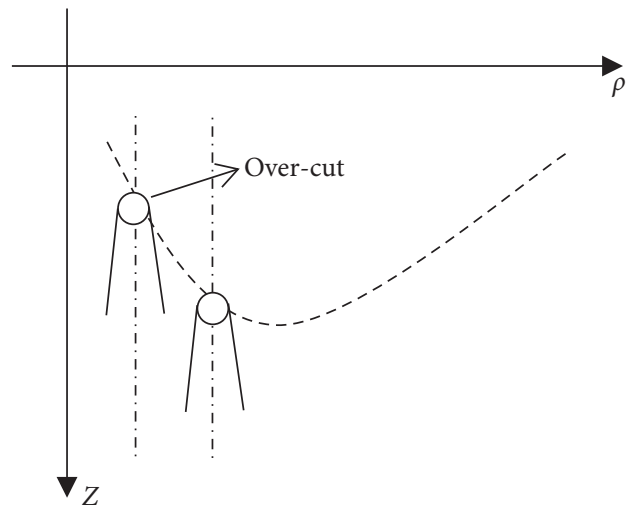

Figure 8: Influences of tool nose radius.

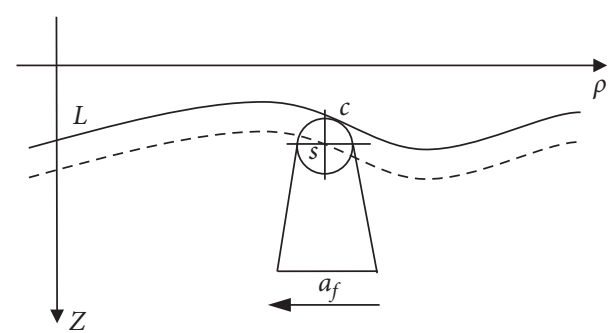

Figure 9: Schematic diagram of tool nose radius compensation.

point according to the feed amount. Meanwhile, the coordinates of the tool contact point $C$ can be derived from the surface equation. Then, the axial coordinate of point $S$ can be calculated according to the coordinates of point $C$. The specific methods are as follows.

The mathematical expression of the freeform surface in cylindrical coordinates can be expressed as follows:

$$
z=f(\rho, \theta) \text {. }
$$

As can be seen from Figure 9, the coordinates of tool location point $S$ can be expressed as follows:

$$
\begin{gathered}
\rho_{s}(\theta)=R-\frac{a_{f} \theta}{2 \pi}, \\
z_{s}(\theta)=f\left(\rho_{s}(\theta)+r \sin a, \theta\right)+r \cos a,
\end{gathered}
$$

where $R$ is the cutting radius ( $\mathrm{mm}), a_{f}$ is the feed rate $(\mathrm{mm} /$ $\mathrm{r}$ ), $r$ is the tool nose radius ( $\mathrm{mm}$ ), and $a$ is the angle between the tangent of the tool contact point $C$ and the $\rho$ axis, which can be obtained from the following implicit equation:

$$
\tan (a)=f^{\prime}\left(\rho_{s}(\theta)+r \sin a, \theta\right)
$$

According to the abovementioned formulas, the coordinates of all tool location point $S$ can be obtained, which completes the tool nose radius compensation.

3.4. Generating Tool Path. After determining the method of selecting discrete points and the method of tool nose radius compensation, we can generate the tool path. The mathematical expression for PAL is available in Section sec 2.2 of this article. We set $M$ as the number of spindle revolutions and $N$ as the sampling number per rotation. Then, the spindle angle can be expressed as follows:

$$
\theta(i, j)=2 \pi\left(i+\frac{j}{N}\right)
$$

where $i$ and $j$ are cycle variables, and $\theta(i, j)$ corresponds to the $j$ th pulse of the $i$ th turn of the spindle. Substituting equation (21) into equations (18) and (20), we can obtain

$$
\begin{gathered}
\rho_{s}(i, j)=R-a_{f}\left(i+\frac{j}{N}\right) \\
\tan a_{p}-f^{\prime}\left(\rho_{s}(i, j)+r \sin a, \theta(i, j)\right)=0 .
\end{gathered}
$$

The value of acorresponding to each cutting point can be obtained by solving equation (23), then the $Z$ coordinate of the tool location point $S$ can be expressed as follows:

$$
z_{s}(i, j)=f\left(\rho_{s}(i, j)+r \sin a(i, j), \theta(i, j)\right)+r \cos a(i, j) .
$$

Using the abovementioned method, the tool path of the PAL surface is generated. Figure 10 is a sketch of spiral tool path generation. In order to display the diagram conveniently, a larger feed speed is adopted. Then, according to the requirements of the $\mathrm{NC}$ system, the coordinates of tool location points are arranged in the form of $\theta-\rho-z$, and the machining program can be obtained.

\section{Experiments and Discussion}

To verify the proposed method, experiments were carried out with Moore's 250UPL single point diamond lathe. The processing mode of the equipment is SSS. The $X$-axis and $Z$ axis of the machine tool are both linear axis and the stroke is $200 \mathrm{~mm}$. The spindle is fixed on the worktable and can move along the $X$-axis. The turning tool moves along the $Z$-axis to achieve vibration cutting. Specific machine layout can be seen in Figure 6. 


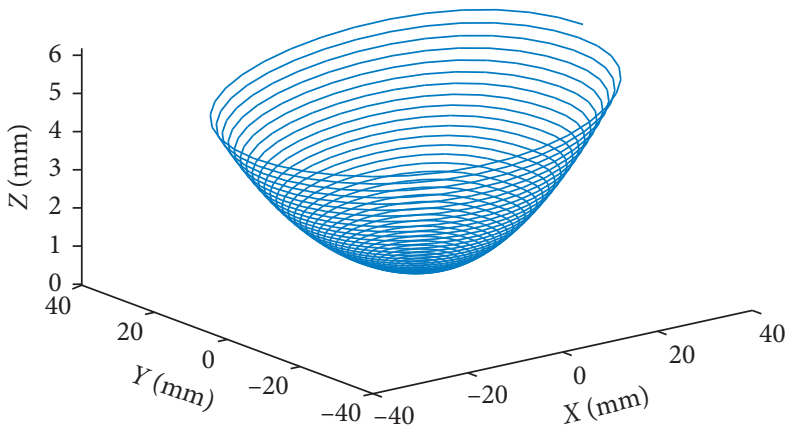

Figure 10: Tool path diagram.

TABLe 2: Machining parameters for PAL.

\begin{tabular}{lccc}
\hline Processing steps & Sampling number & Feed rate $(\mathrm{mm} / \mathrm{r})$ & Tool nose radius $(\mathrm{mm})$ \\
\hline Roughing & 36 & 0.1 & 0.8 \\
Finishing & 180 & 0.01 & 0.8 \\
\hline
\end{tabular}

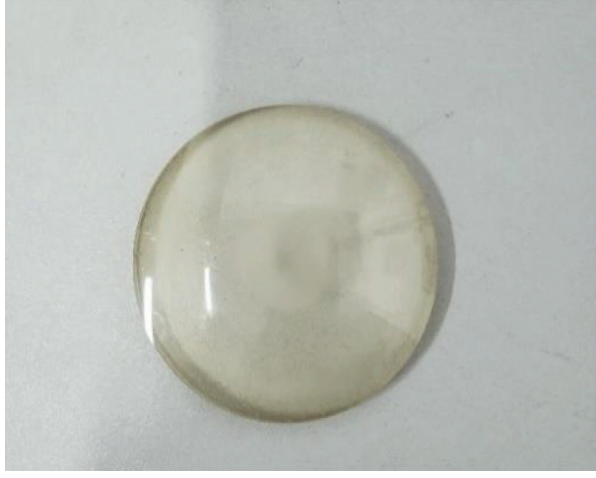

(a)

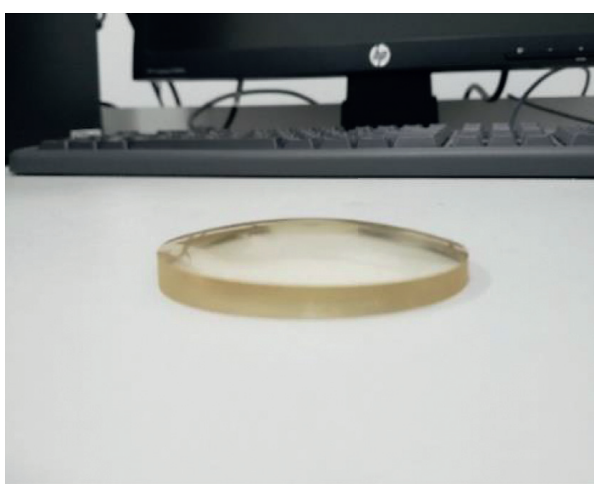

(b)

Figure 11: Photos of the PAL.
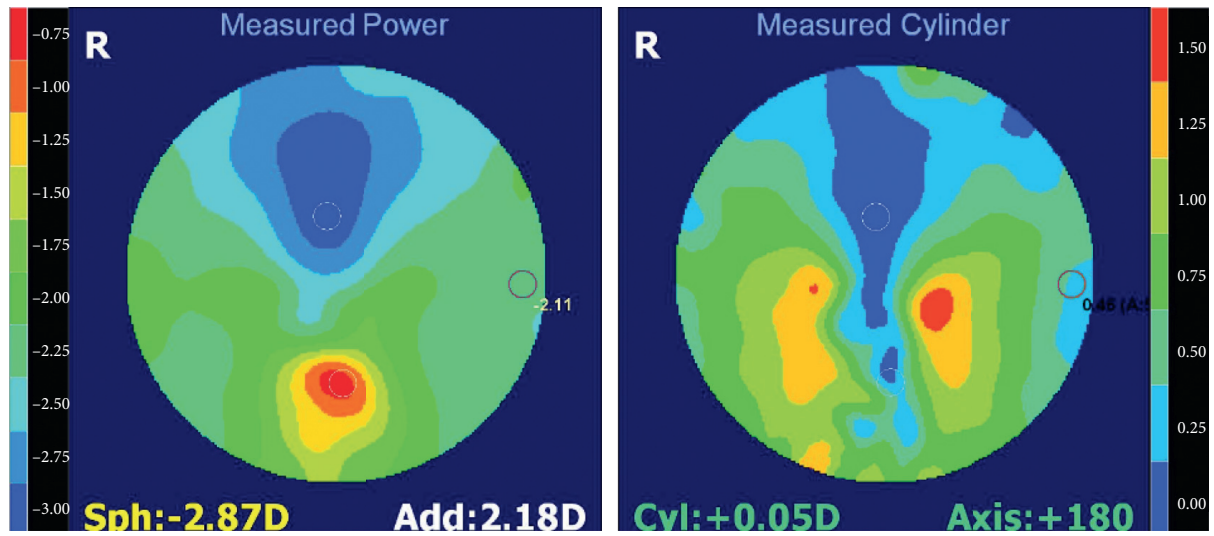

Figure 12: Power and astigmatism distribution of the lens.

Because the surface of precision components cannot be pressurized, the workpiece is fixed on the machine by the way of sucker and bonding. The material of the workpiece is resin with a diameter of $70 \mathrm{~mm}$. The specific optical design parameters are shown in Table 1. Machining process is divided into roughing and finishing, and processing parameters can be seen in Table 2 . The processing results are shown in Figure 11.

The surface roughness was measured by the Dektak XT probe surface profiler. In order to make the measurement 
more accurate, the surface roughness was measured in four directions $(0,90,180,270$ degrees), twice in each direction, and then the average value was calculated. The sampling length is $0.8 \mathrm{~mm}$ and the evaluation length is $4 \mathrm{~mm}$. The average roughness $(\mathrm{Ra})$ obtained was $0.087 \mu \mathrm{m}$. The power and astigmatism (or called cylinder) distribution of the lens were measured with the Rotlex freeform verifier, as shown in Figure 12. It is less than $0.18 \mathrm{D}$ that the different of the addition power between the designed and manufactured PAL. The results show that the power of the far- and nearsighted zone of the lens changes slowly, the transition zone (progressive corridor) changes smoothly and gradually, and the astigmatism mainly concentrates on both sides of the lens, which meets the design requirements.

\section{Conclusions}

This paper presents a PAL design method based on mathematical principles, which is different from the freeform surface model formed by spline interpolation to obtain more accurate calculation data. Based on the PAL design method, an optimized tool path generation method which can avoid unnecessary vibration of machine tool is proposed. The surface of PAL is obtained by turning experiment, and the rationality of optical design is verified by the Rotlex freeform verifier. Some conclusions are summarized as follows:

(1) According to the method described in Section 2, different parameters can be selected for individualized design of lenses. The design parameters can be selected including the values of $m$ and $l$, the length of light adding channel, the type of orthogonal contour curve, and the power of far-view zone and near-view zone. The combination of different parameters can meet the different needs of wearers.

(2) The tool path generation method described in this paper is suitable for both FTS and SSS technology. It can provide reference for diamond turning of optical freeform surface components. A more stable tool nose radius compensation method is adopted. It can make the $X$-slide feed uniformly throughout the processing, avoid unnecessary acceleration, and obtain better surface quality.

(3) The designed PAL was fabricated by a single point diamond lathe. The results show that the optical distribution of the lens is consistent with the design, and the power error is within an acceptable range. The surface quality is good and the roughness is $0.087 \mu \mathrm{m}$.

\section{Data Availability}

The data used to support the findings of this study are available from the corresponding author upon request.

\section{Conflicts of Interest}

The authors declare that there are no conflicts of interest regarding the publication of this paper.

\section{Acknowledgments}

This work was supported by the Shandong Provincial Natural Science Foundation (Grant no. ZR2019MEE086); the Major Technological Innovation Project of High-end Manufacturing Equipment in Shandong Science and Technology Department (Grant no. 2017CXGC0911); and the Shandong Taishan Leadership Training Project (Grant no. 2016GRC3205).

\section{References}

[1] K. Garrard, T. Bruegge, J. Hoffman, T. Dow, and A. Sohn, "Design tools for freeform optics," in Proceedings of the Current Developments in Lens Design and Optical Engineering VI, vol. 5874, pp. 1-11, SPIE, San Diego, CA, USA, August 2005.

[2] X. Jiang, P. Scott, and D. Whitehouse, "Freeform surface characterisation-a fresh strategy," CIRP Annals, vol. 56, no. 1, pp. 553-556, 2007.

[3] L. B. Kong, C. F. Cheung, J. B. Jiang, S. To, and W. B. Lee, "Characterization of freeform optics in automotive lighting systems using an optical-geometrical feature based method," Optik, vol. 122, no. 4, pp. 358-363, 2011.

[4] X. Jiang, P. J. Scott, D. J. Whitehouse, and L. Blunt, "Paradigm shifts in surface metrology. Part II. The current shift," Proceedings of the Royal Society A: Mathematical, Physical and Engineering Sciences, vol. 463, no. 2085, pp. 2071-2099, 2007.

[5] W. Zhu and A. Beaucamp, "Zernike mapping of optimum dwell time in deterministic fabrication of freeform optics," Optics Express, vol. 27, no. 20, pp. 28692-28706, 2019.

[6] L. Dick, S. Risse, and A. Tünnermann, "Process influences and correction possibilities for high precision injection molded freeform optics," Advanced Optical Technologies, vol. 5, pp. 277-287, 2016.

[7] J. Xie, J. H. Zheng, R. M. Zhou, and B. Lin, "Dispersed grinding wheel profiles for accurate freeform surfaces," International Journal of Machine Tools and Manufacture, vol. 51, no. 6, pp. 536-542, 2011.

[8] M. N. Cheng, C. F. Cheung, W. B. Lee, S. To, and L. B. Kong, "Theoretical and experimental analysis of nano-surface generation in ultra-precision raster milling," International Journal of Machine Tools and Manufacture, vol. 48, no. 10, pp. 1090-1102, 2008.

[9] L. B. Kong and C. F. Cheung, "Prediction of surface generation in ultra-precision raster milling of optical freeform surfaces using an integrated kinematics error model," Advances in Engineering Software, vol. 45, no. 1, pp. 124-136, 2012.

[10] Z. Q. Yin, Y. F. Dai, S. Y. Li, C. L. Guan, and G. P. Tie, "Fabrication of off-axis aspheric surfaces using a slow tool servo," International Journal of Machine Tools and Manufacture, vol. 51, no. 5, pp. 404-410, 2011.

[11] F. Z. Fang, X. D. Zhang, and X. T. Hu, "Cylindrical coordinate machining of optical freeform surfaces," Optics Express, vol. 16, no. 10, pp. 7323-7329, 2008.

[12] F. Tian, Z. Yin, and S. Li, "Fast tool servo diamond turning of optical freeform surfaces for rear-view mirrors," The International Journal of Advanced Manufacturing Technology, vol. 80, no. 9-12, pp. 1759-1765, 2015.

[13] Q. Liu, "Study on freeform optical surfaces diamond turning assisted by fast tool servo," Doctor thesis, Jilin University, Changchun, China, 2012. 
[14] S. S. Douglas, "A machining system for turning non-axisymmetric surface," Doctor thesis, University of Tennessee, Knoxville, TN, USA, 1983.

[15] M. Kang, J. Yang, X. S. Wang, and X. Chen, "Study on the variational-difference-based design and slow tool servo turning of progressive addition lenses," AIME, vol. 10, pp. 1-12, 2018.

[16] W.-Y. Hsu, Y.-L. Liu, Y.-C. Cheng, C.-H. Kuo, C.-C. Chen, and G.-D. Su, "Design, fabrication, and metrology of ultraprecision optical freeform surface for progressive addition lens with B-spline description," The International Journal of Advanced Manufacturing Technology, vol. 63, no. 1-4, pp. 225-233, 2012.

[17] J. T. Winthrop, "Progressive addition spectacle lenses," US Patent 4861153, 1989.

[18] J. T. Winthrop, "Hard/soft superposition progressive lens design," US Patent 5726734, 1998.

[19] T. Steele, "Progressive addition power," US Patent 6776486, 2004.

[20] B. F. Maitenaz, "Ophthalmic lenses with a progressively varying focal power," US Patent 3687528, 1972.

[21] J. T. Winthrop, "Progressive addition spectacle lens," US Patent 5123725, 1992.

[22] Y. Tang, Q. Wu, X. Chen, and H. Zhang, "A personalized design for progressive addition lenses," Optics Express, vol. 25, no. 23 , pp. $28100-28111,2017$.

[23] D. Li, B. Wang, Z. Qiao, and X. Jiang, "Ultraprecision machining of microlens arrays with integrated on-machine surface metrology," Optics Express, vol. 27, no. 1, pp. 212-224, 2019.

[24] D. Li, Z. Qiao, K. Walton et al., "Theoretical and experimental investigation of surface topography generation in slow tool servo ultra-precision machining of freeform surfaces," $M a$ terials, vol. 11, no. 12, p. 2566, 2018.

[25] L. B. Kong, C. F. Cheung, and T. C. Kwok, "Theoretical and experimental analysis of the effect of error motions on surface generation in fast tool servo machining," Precision Engineering, vol. 38, no. 2, pp. 428-438, 2014. 